\title{
Porphyrin Langmuir-Blodgett Thin Film for Organic Vapor Detection
}

\author{
Rifat Çapan \\ Physics Department, Science Faculty, Balıkesir University, Balıkesir 10145, Turkey
}

\begin{abstract}
The detection of volatile organic compounds (VOC's) is a very important task due to stringent environmental standards and regulations on VOC's in many countries. They are highly toxic and may cause metabolic diseases, genetic disorders, acute, chronic or long-term effects and cancer. In order to control the air quality and human health respect to standards and regulations gas sensors are widely used to measure the level of VOCs. The performance of the gas sensing devices mostly depends on the performance of the sensing materials which depends on their chemical structures. Organic materials are widely studied in the field of VOC's sensor application due to their low cost, physical and chemical properties. Porphyrin molecule was deposited as an Langmuir-Blodgett thin film onto gold substrates for the study of sensor properties using benzene, toluene, ethyl benzene and xylene. Surface plasmon resonance technique was employed for the characterization of these LB films. A high quality and uniform Langmuir monolayer from the water surface can be transferred onto a gold coated substrate with a transfer ratio of over $90 \%$. For the sensing application towards benzene vapor, this LB film yields a fast and almost fully reversible response to benzene in few seconds.
\end{abstract}

Keywords: Porphyrin, vapor sensing, langmuir-blodgett, surface plasmon resonance.

\section{Introduction}

A porphyrin molecule has a large ring molecule consisting of 4 pyrroles, which are smaller rings made from 4 carbons and 1 nitrogen. These pyrrole molecules are connected together through a series of single and double bonds which forms the molecule into a large ring. The ring is flat in space, and the distribution of electrons is fairly equal around the circumference of the ring. For this reason, a porphyrin is considered an aromatic compound and it is very stable. Several hydrophilic and hydrophobic functional groups or metals can easily bound to these rings to synthesis an LB film material. Porphyrins are known to be excellent Langmuir and Langmuir-Blodgett film forming materials and they are used as specific ligands for analytical chemistry, sensor techniques, and medical diagnostics, since a number of synthesis methods allow flexible variation

Corresponding author: Rifat Çapan, Ph.D, professor, research fields: nanotechnology, gas sensor application, physical properties of organic thin film. E-mail: rcapan@balikesir.edu.tr. of their ring size, they can be functionalized with a wide range of functional groups, and have structural characteristics such as high porosity and good physical, chemical and thermal stability [1]. Chemical sensors based on relatively low cost synthesis porphyrins were designed to detect various inorganic gases [2], explosive vapors [3], and volatile organic compounds (VOC's) [4-6] because of their flexible structure, as well as their wide range of physical and chemical properties that can be tailored by changing their compositions.

The monitoring and detection of VOC's has become a serious aspect to consider because the need to control air quality has become an environmentally important issue. The construction of gas and VOC sensors will allow the rapid detection of harmful or environmentally damaging vapors without the need for techniques such as gas chromatography or mass spectrometry which often require expensive equipment and highly trained laboratory personnel. Improving the performance of gas sensing devices mostly depends on the sensitivity and selectivity of 
the sensing materials. It is well known that when a gas molecule is adsorbed onto the surface of an organic material, the physicochemical properties, including the structural, electrical, and optical properties, of this sensing material can change. This has led to intensive research into new materials with the ability to bind, detect and identify organic vapors. The chief difficulty in gas identification continues to be the fabrication of stable sensors with a high sensitivity and selectivity towards the substance to be detected. In the last decade the use of organic materials as gas sensing materials has increased because of their simple and low cost synthesis, wide range of physical and chemical properties that can be tailored by changing their composition. Organic materials have several potential applications in the field of physics, chemistry, biology, material, environmental science and are widely used to produce highly uniform thin films using spin coating, self assembly, Langmuir-Blodgett (LB) thin film technique [7, 8]. They are suitable materials for the detection of several toxic gases or dangerous organic vapors. Because of the sensitivity of porphyrin to organic vapors and their excellent LB film forming ability, we have investigated in depth the use of these materials to sense VOCs. Previous work has demonstrated sensitivity of porphyrin LB films to VOC's [9]. A number of other authors have also carried out studies on these systems, for example SPR could be used to measure the adsorption of a range of organic compounds onto porphyrin LB thin films [10-12].

In here, a porphyrin material incorporating $\mathrm{Zn}$ is selected to prepare LB thin films and to investigate their sensing properties of dangerous organic vapors. In here, several organic materials are selected to prepare thin organic films and to investigate their sensing properties of dangerous organic vapors. Surface Plasmon Resonance (SPR) optical technique is employed to monitor the deposition process of the thin film and organic vapor sensing properties. Our results showed that this selected material could have potential applications in the field of sensor industry.

\section{Experimental Details}

A computer controlled NIMA 622 alternate LB trough was employed to study the molecular behavior of porphyrin molecule at the air-water interface and to produce LB films onto $50 \mathrm{~nm}$ thick gold coated glass substrates. Before each experiment, the barriers and the Teflon trough of the LB film system were rinsed with ultrapure water $(18.2 \mathrm{M} \Omega \mathrm{cm})$ after being cleaned with chloroform. The surface pressure was measured by using a Wilhelmy balance, equipped with a strip of chromatography paper suspended at the air-water interface at $20^{\circ} \mathrm{C}$ which was controlled using Lauda Ecoline RE204 model temperature control unit. Porphyrin molecule was dissolved in chloroform with a concentration of $0.2 \mathrm{mg} \mathrm{mL}^{-1}$ and was subsequently spread onto ultrapure water subphase at $\mathrm{pH} 6$. Solutions were spread by a Hamilton microliter syringe onto the subphase solution by distributing the droplets over the entire trough area. A time period of $20 \mathrm{~min}$ was allowed for the solvent to evaporate before the area enclosed by the barriers was reduced. The pressure-area $(\pi-\mathrm{A})$ isotherm graph was determined with the accuracy of $0.1 \mathrm{mN} \mathrm{m}^{-1}$. This graph was recorded as a function of surface area using the compression speed of barriers at a value of 172 $\mathrm{mm}^{2} \mathrm{~min}^{-1}$. The floating monolayer at the air-water interface was found to be stable at a surface pressure of $22.5 \mathrm{mN} \mathrm{m}^{-1}$; therefore, this surface pressure value was selected for the LB film deposition procedure. Y-type LB deposition mode and a vertical dipping procedure was performed at the selected surface pressure with a speed of $10 \mathrm{~mm} \mathrm{~min}^{-1}$ for both the down and up strokes. LB film samples were dried for 5 min after each up stroke.

A Kretschmann type Surface Plasmon Resonance Spectrometer with a low power laser diode $(633 \mathrm{~nm})$ light source was employed for SPR measurements. A glass prism $(\mathrm{n}=1.515)$ mounted onto a holder is available for measurement in liquid or in air. The glass 
slides had the dimensions of $20 \times 20 \mathrm{~mm}$ and the total thickness was $1 \mathrm{~mm}$ are coated on the top by a $50 \mathrm{~nm}$ thin gold layer. After each deposition cycle, the porphyrin LB film sample was dried for half an hour and the SPR curve was monitored using SPR measurement system. This system was used for the confirmation of the reproducibility of LB film multilayers using the relationship between the angle changes against the deposited thickness, which should depend on the number of layers in the LB film. Benzene, ethyl benzene, toluene and xylene (99\% Aldrich) were used without further purification as organic vapors for the detection performances. All measurements were taken in dry air condition in a small gas cell which could eliminate the effect of water vapor on the response properties of LB film sample. A special flow cell from transparent plastic, compatible for vapor or gas measurements was constructed to study the kinetic response of porphyrin LB film on exposure to organic vapors by measuring the reflected light intensity changes. The cell has two channels, with inlet and outlet and VOC injections were performed with a syringe. The SPR system is completely controlled by computer, the measurements and data acquisition as well as controlling to measurement and data presentation. The response was recorded as a function of time when the sample was periodically exposed to the organic vapors for at least 2 min and was then allowed to recover after injection of dry air.

\section{Result and Discussions}

Fig. 2a shows the experimental SPR curves with the shift of the photodetector response (intensity of reflected light) as a function of angle of bare gold and increasing number of layers between 2-8 layers of porphyrin LB films. The SPR curves of porphyrin LB films show that the different thicknesses are changed to larger angles with the number of layers is increased by using the curve of uncoated gold as a reference. Fig. $2 \mathrm{~b}$ displays the peak shifts $(\Delta \theta)$ seen in the angular scans of the plasmon resonance curves of the LB film multilayer assemblies relative to bare gold, which increased linearly with the number of layers. This linear relationship suggests that an equal mass per unit area is deposited onto the gold-coated glass substrate during the transfer of LB film layers.

It is important to understand the response properties

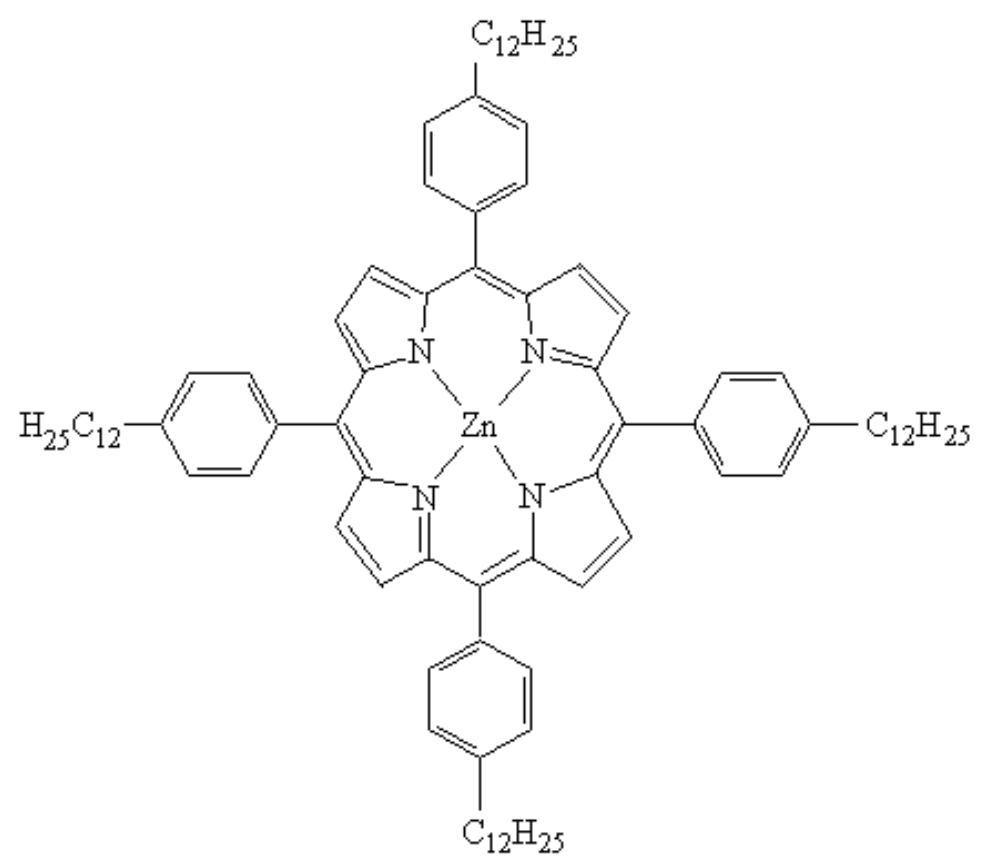

Fig. 1 Chemical structure of porphyrin. 


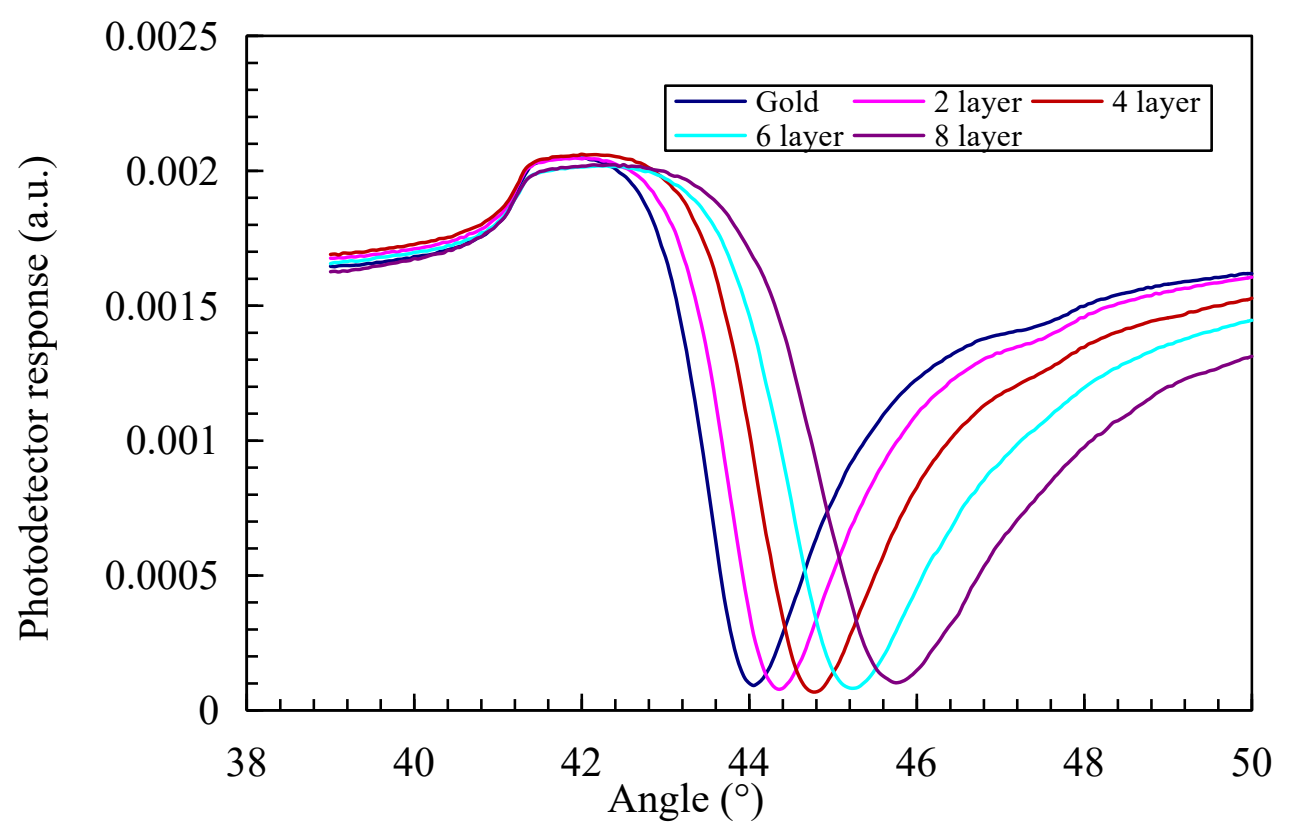

(a)

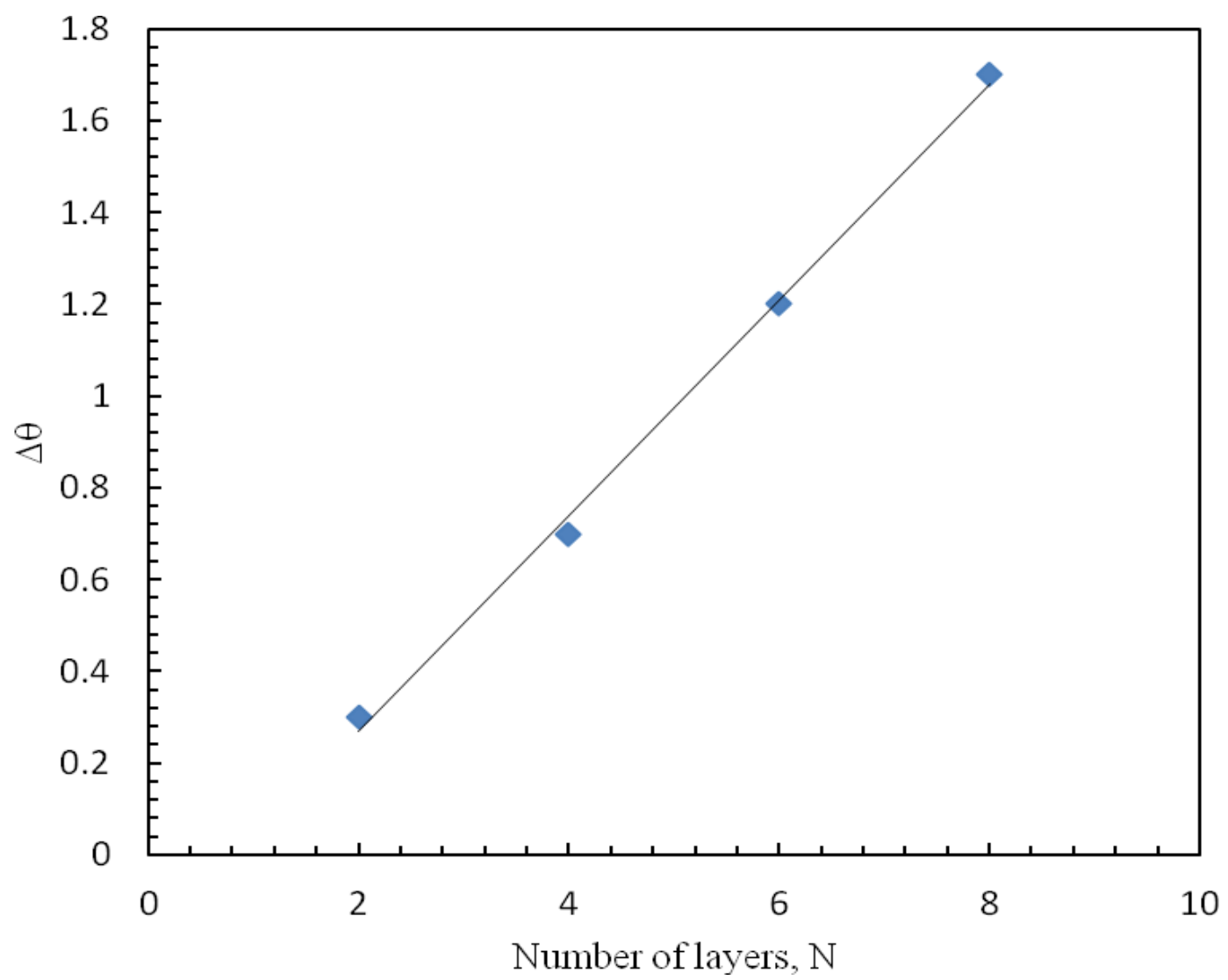

(b)

Fig. 2 a) SPR curves for the porphyrin LB films with different numbers of layers b) thickness of the thin films as a function of the number of layers. 
between the porphyrin LB film and organic vapors to allow for the detection and identification of organic vapor for application as room temperature vapor sensors. In the SPR vapor sensing arrangement, usually, a photodiode operates as a detector providing an output voltage proportional incident light intensity. The measurement angle is fixed at a point on the steepest part of the resonance curve and the photodiode output is a monitored as a function of time. This is called a kinetic measurement. The kinetic responses of the porphyrin $\mathrm{LB}$ film to the benzene, ethyl benzene, toluene and xylene vapors is given in Figs. 3-6 by measuring the reflected light intensity as a function of time for $2 \mathrm{~min}$, followed by injection of dry air for a further $2 \mathrm{~min}$ period.

Fig. 3a shows a typical SPR curve obtained for the porphyrin LB film before and after the exposure of benzene vapor. It is clear that a large shift $\theta$ in the SPR minimum occurred due to the adsorption of saturated benzene vapor on the porphyrin film. The shift of SPR minimum is usually explained in terms of an increase in refractive index of the sensitive layer and change in the film thickness due to the film swelling [13]. The refractive index of benzene is 1.5011 , a value close to that of the porphyrin film and the effect of benzene adsorption on the refractive index is not expected to be significant. It is therefore believed that the resonance shift is primarily caused by the change in the film thickness due to swelling. On injection of clean air into the gas cell, the recovery is found to be almost complete. The response of porphyrin to benzene is, therefore, fast and reversible.

Kinetic response of porphyrin $\mathrm{LB}$ film to the benzene vapor was recorded by measuring the reflectivity at a fixed angle $\theta=44.5^{\circ}$. Fig. 3b shows the variation of the intensity signal as a function of time when porphyrin LB film was periodically exposed to benzene vapor for 2 min followed by the injection of dry air for a further 2 min period. The first stage in VOC analysis is to flush a reference gas (dry air) through the sensor to obtain a baseline. When the LB film sensor material is exposed to the organic vapor, this causes changes in the output signal until the sensor reaches a steady-state. The vapor is finally flushed out of the sensor using the dry air and the sensor response returns back to its baseline. The time during which the sensor is exposed to the vapor is called "the response time" while the time it takes the sensor to return to its baseline resistance is "the recovery time". Here, porphyrin LB film shows a fast response to benzene as well as a fine degree of recovery, with response and recovery times typically of a few seconds.

Similar investigations in Figs. 4-6 were carried out for other ethyl benzene, toluene, and xylene at the nominally same concentrations. Values of $\theta$ which indicates the degree of response are measured with an accuracy of $0.01^{\circ}$. The results of observations were summarized that porphyrin LB film is reasonably selective for benzene. Benzene vapor shows a higher response that toluene vapor; however both of them have benzene rings. It is well known that the number of adsorbed benzene vapors is higher than toluene vapors because benzene is more volatile, has a lower molar volume and a relatively high viscosity parameter, indicating that benzene molecules are more mobile than the toluene vapors and penetrate easily into the porphyrin LB film structure. ${ }^{[14]}$ Benzene is also a nonpolar solvent and toluene, ethyl benzene and xylene are expected to show stronger solvation than benzene. Dissociated solvent ions may bind a shell of ions, increasing the effective radius for dissolving in the material structure [15]. This dissociation may not occur for benzene, producing the highest SPR response. 


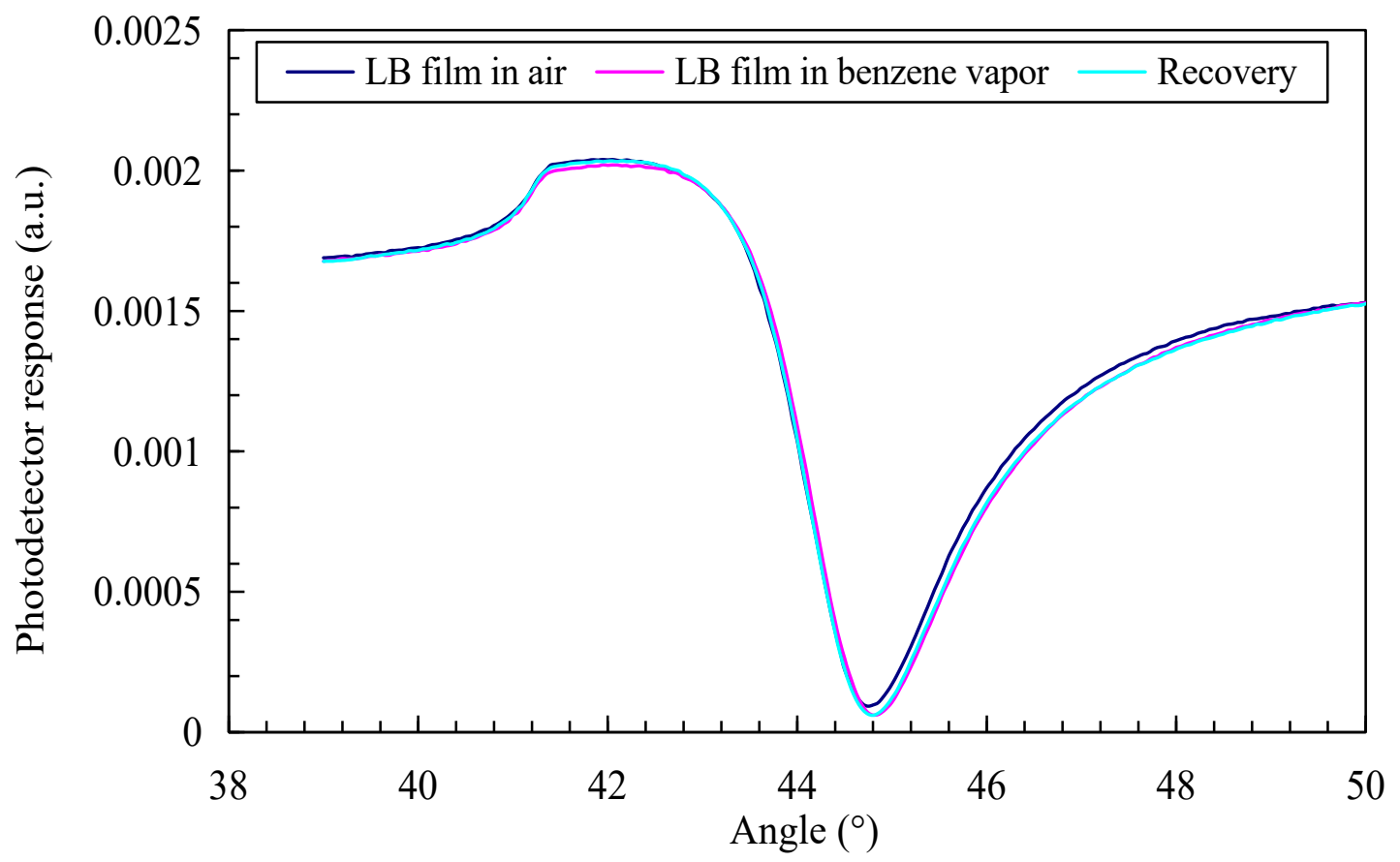

(a)

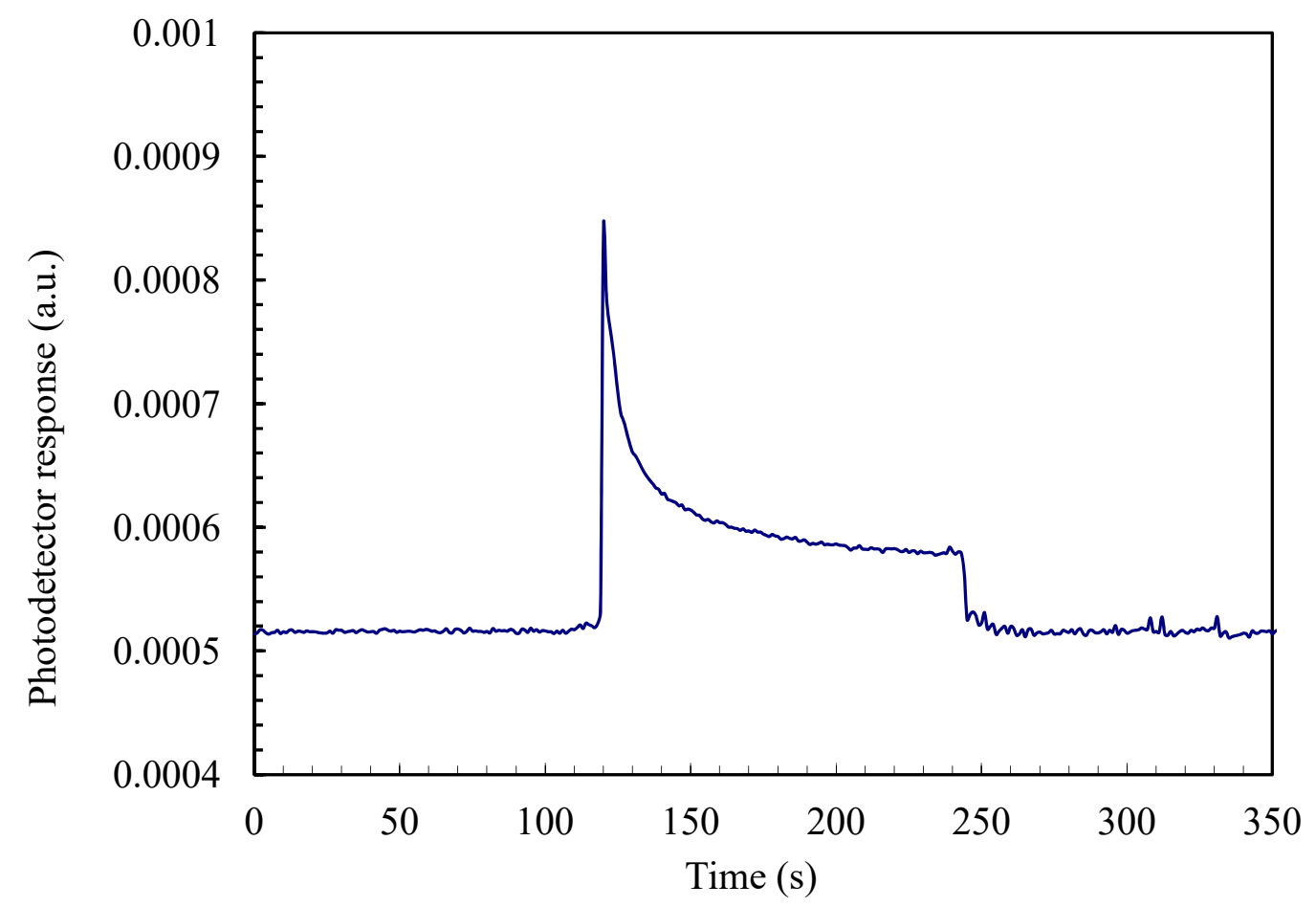

(b)

Fig. 3 Kinetic response of sensor coated with porphyrin to benzene a) SPR curve b) time dependent. 


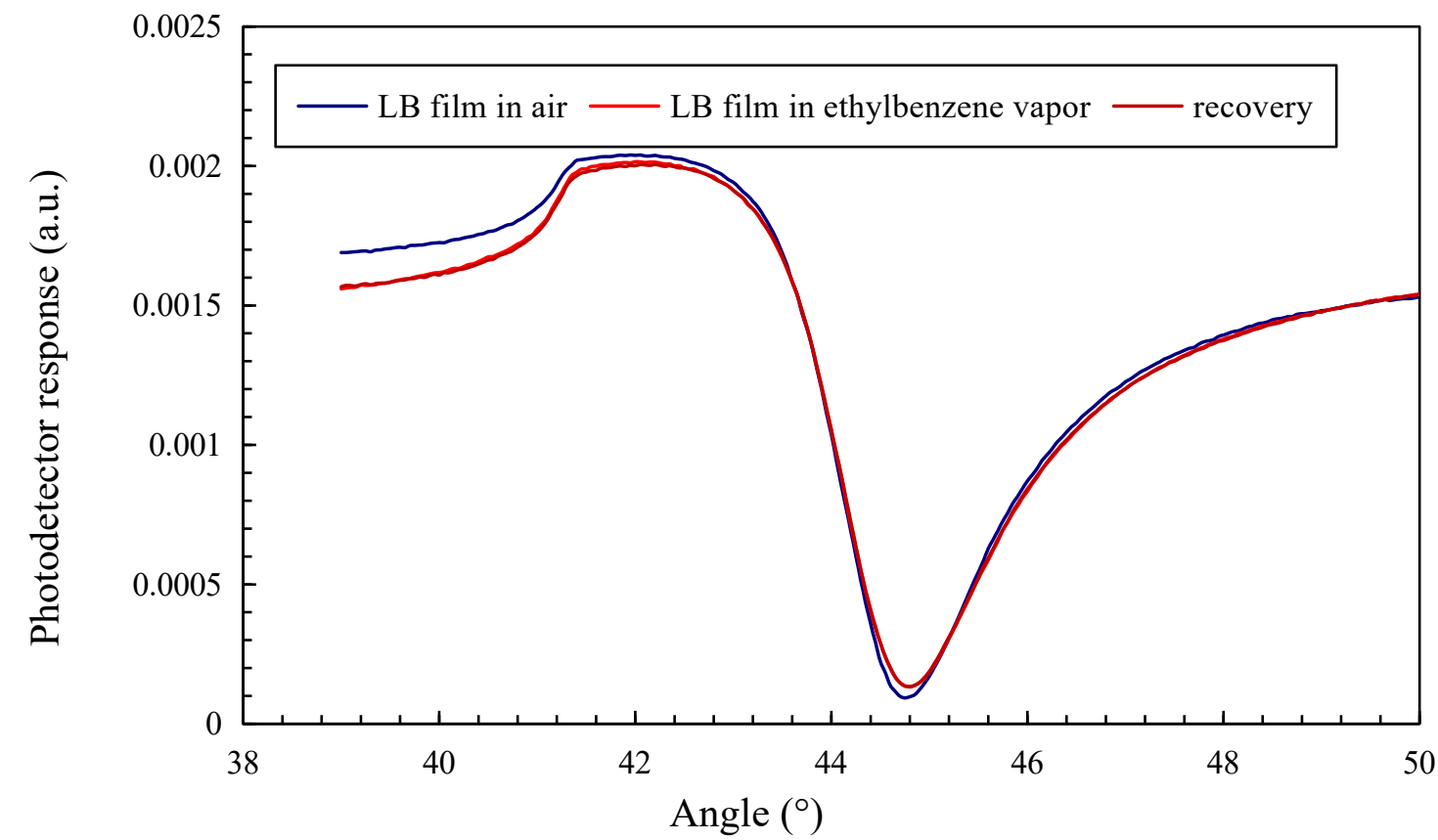

(a)

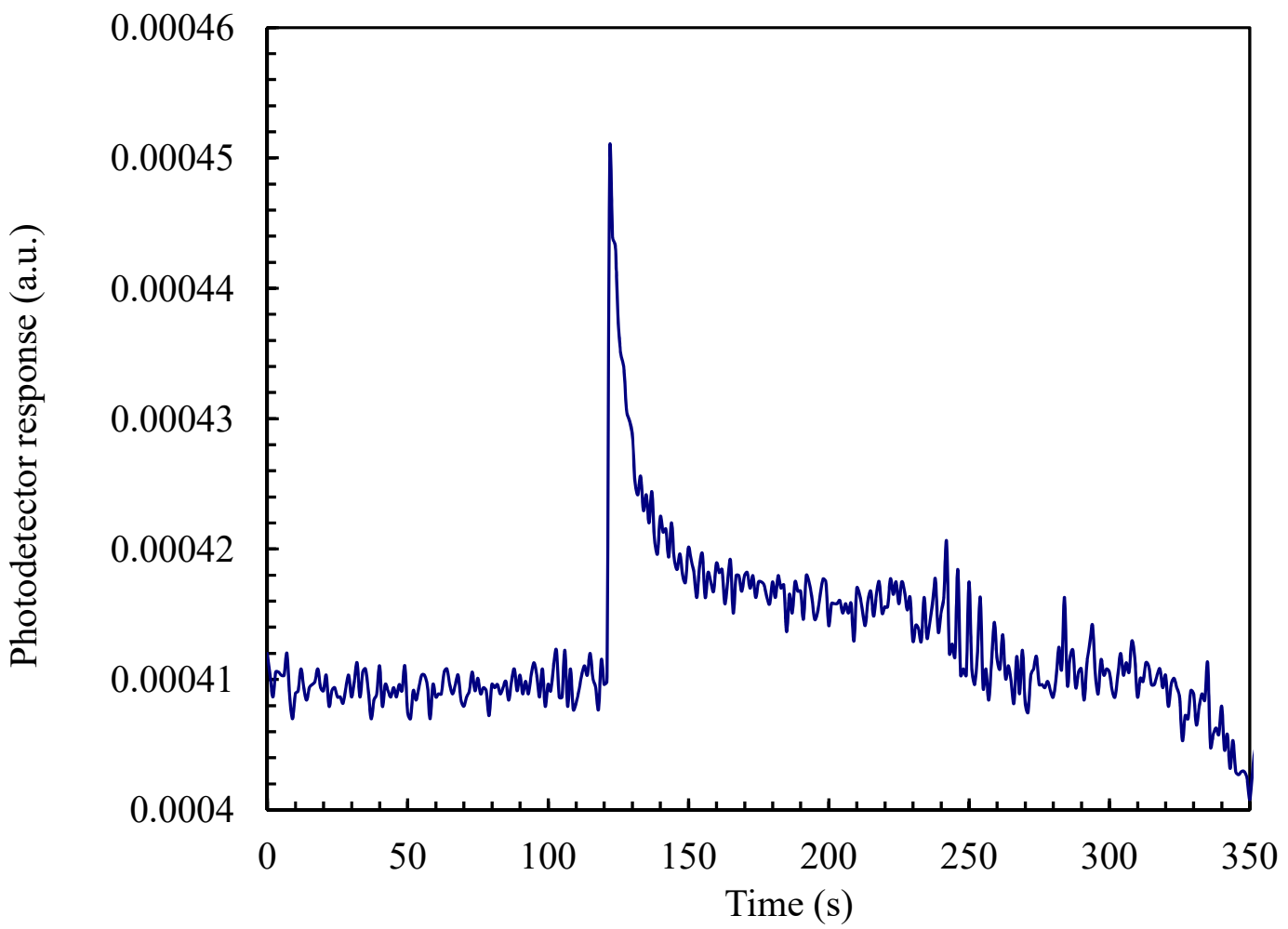

(b)

Fig. 4 Kinetic response of sensor coated with porphyrin to ethyl benzene a) SPR curve b) time dependent. 


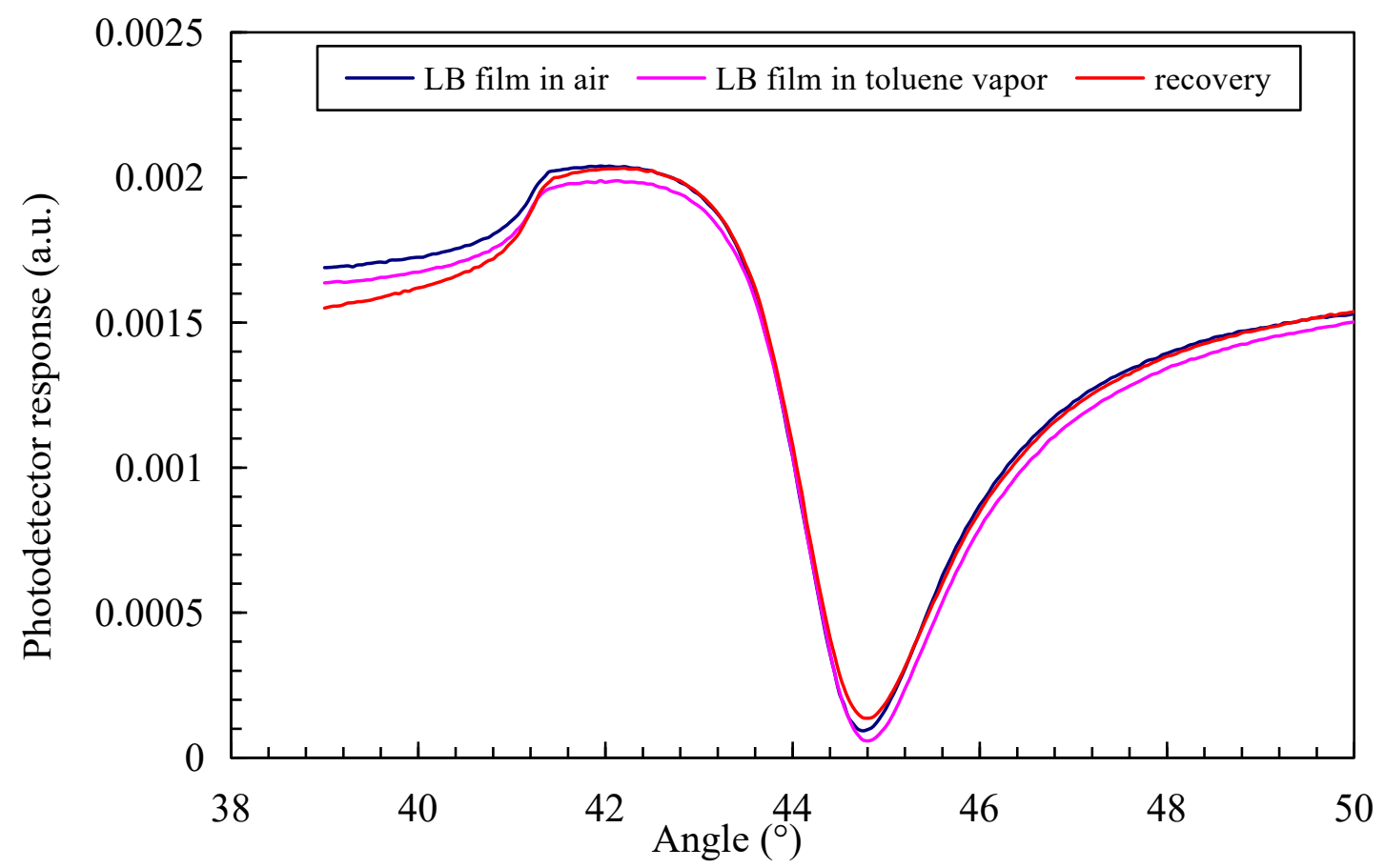

(a)

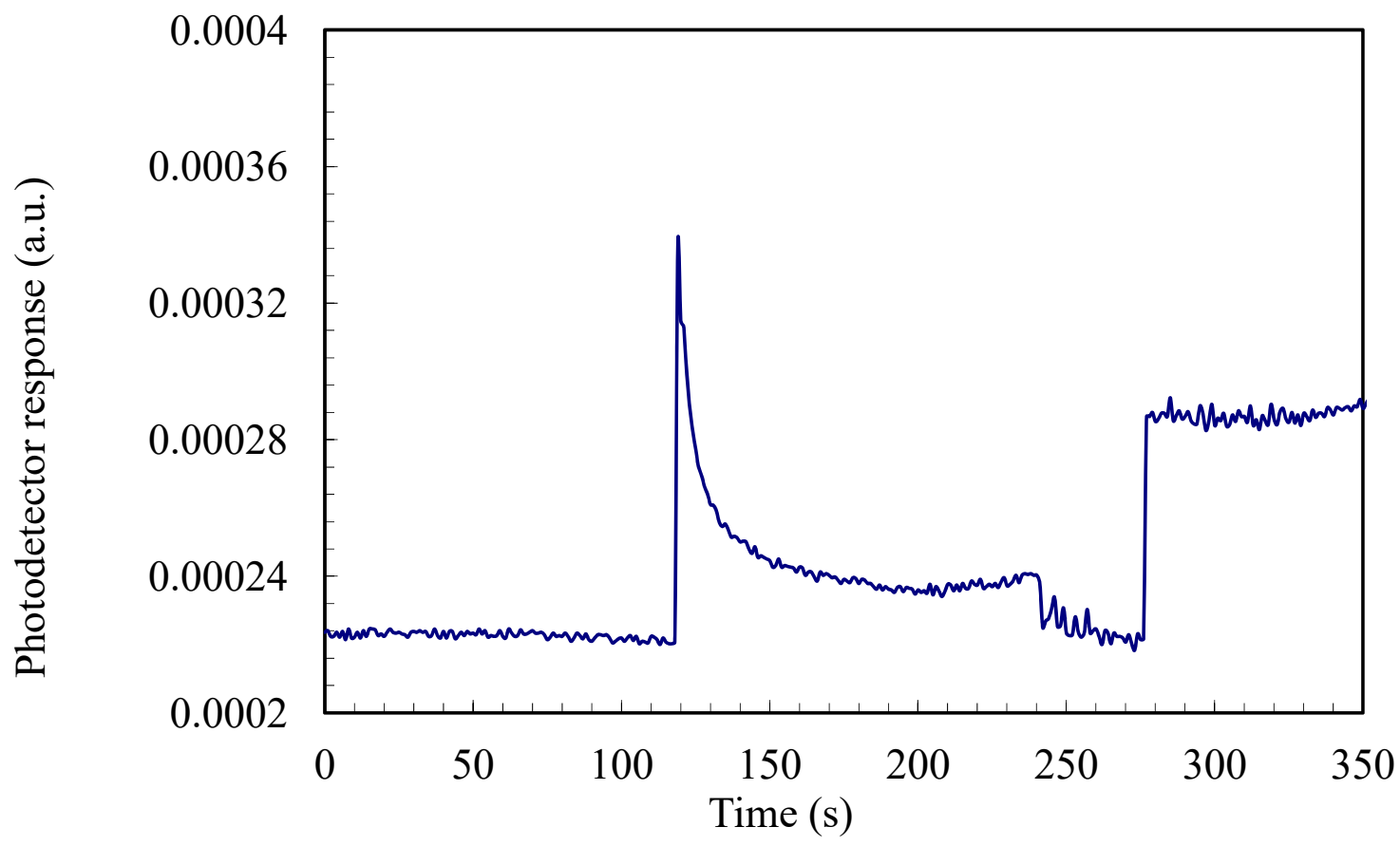

(b)

Fig. 5 Kinetic response of sensor coated with porphyrin to toluene a) SPR curve b) time dependent. 


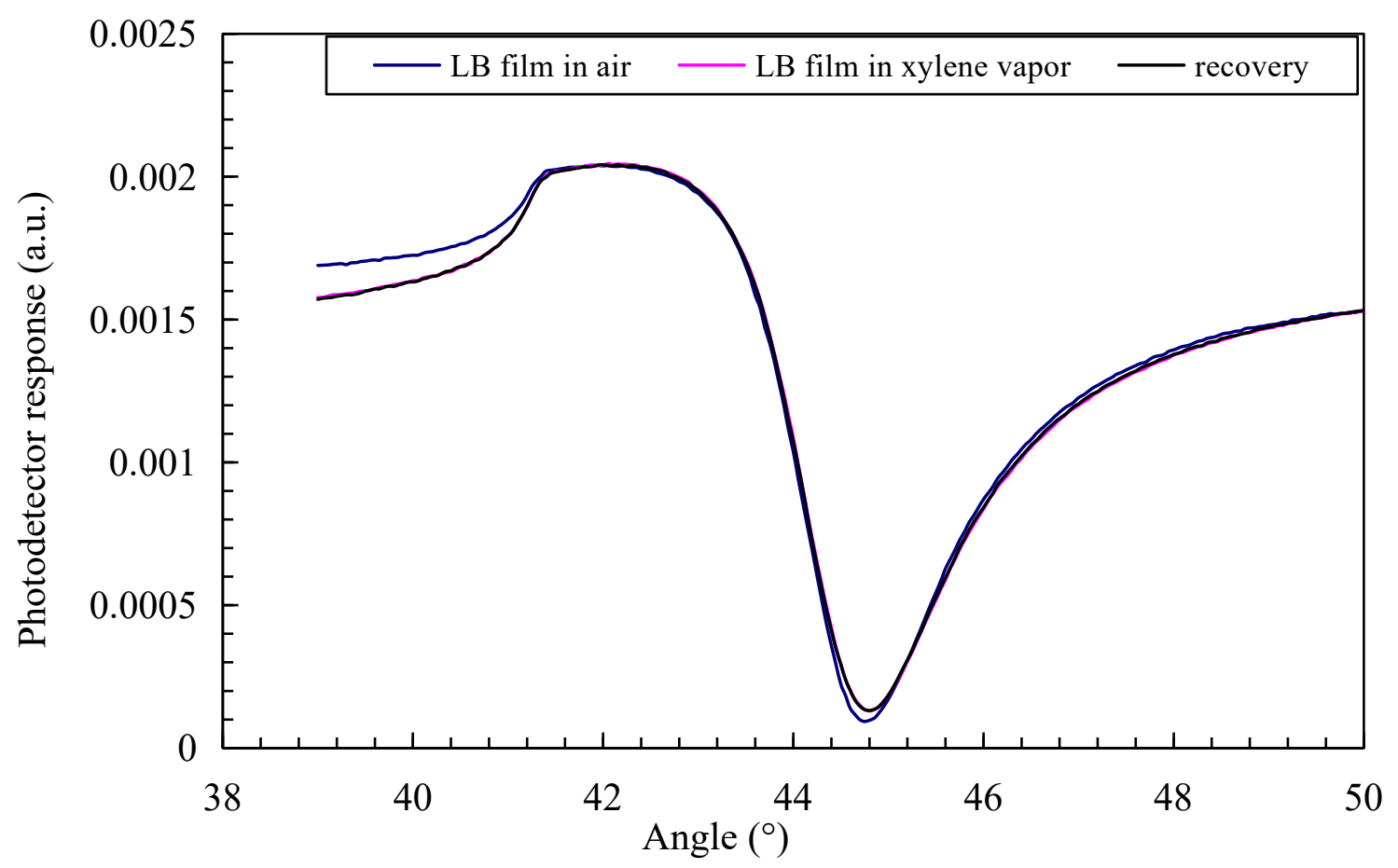

(a)

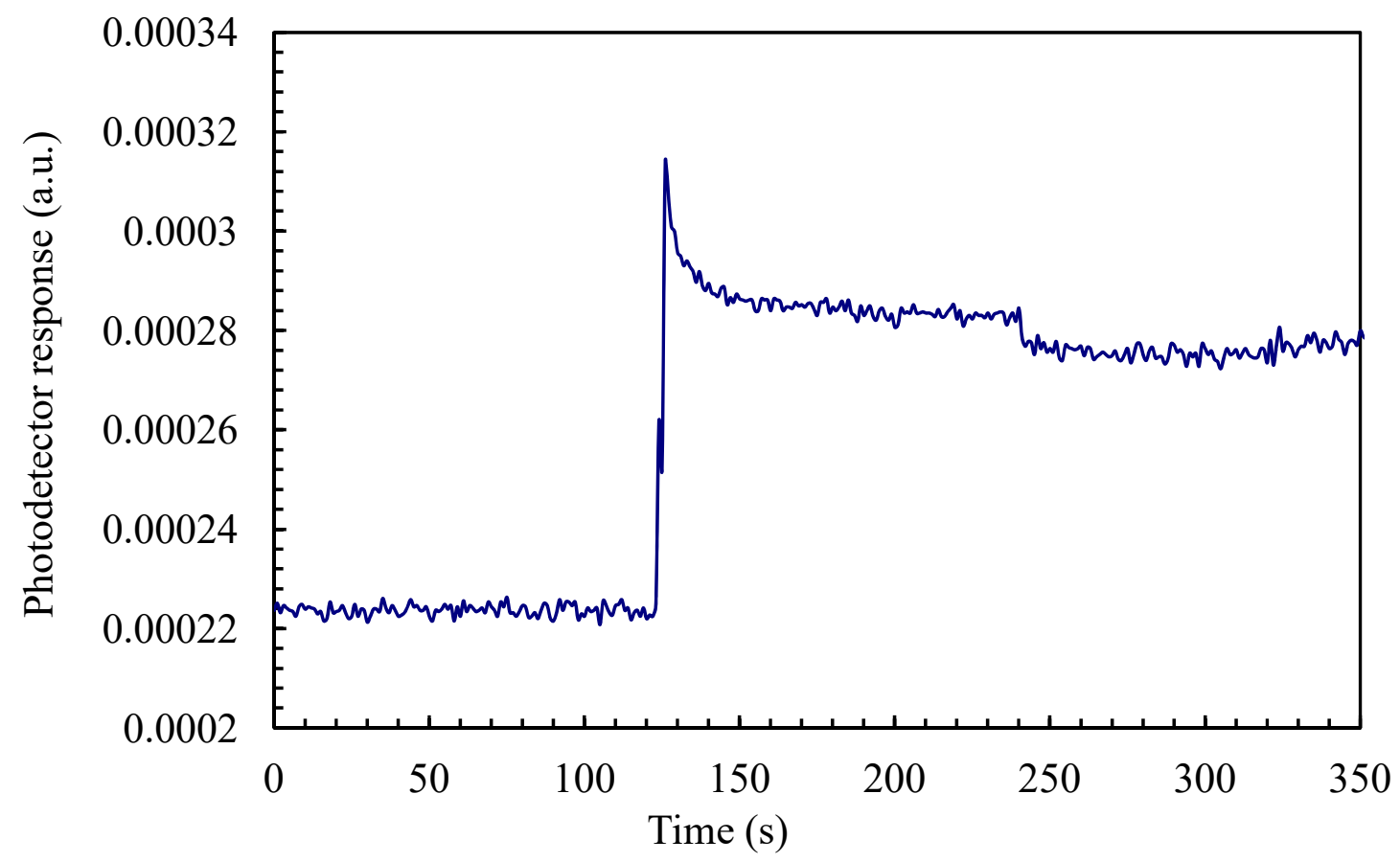

(b)

Fig. 6 Kinetic response of sensor coated with porphyrin to xylene a) SPR curve b) time dependent. 


\section{Conclusion}

Porphyrin material is suitable LB thin film material for the application of VOC's in the nanoscale. SPR deposition measurements indicate a uniform LB monolayer is transferred with a transfer ratio of 0.90 from water surface to a gold coated glass substrate. Porphyrin LB gas sensor shows significantly sensitive and selective to benzene vapor than others. The response of the film to benzene is fast, large, reproducible and had a short recovery time, typically of a few seconds. These results demonstrate the possibilities for the development of optical sensors for porphyrin films.

\section{References}

[1] Day, N. U., Wamsera, C. C., and Walter, M. G. 2015. "Porphyrin Polymers and Organic Frameworks." Polym. Int. 64 (7): 833-857.

[2] Giancane, G., and Valli, L. 2012. "State of Art in Porphyrin Langmuir-Blodgett Films as Chemical Sensors." Adv. Colloid Interface Sci. 171: 17-35.

[3] Guilléna, M. G., Gámez, F., Roales, J., Lopes-Costa, T., Pinto, S. M. A., Calvete, M. J. F., Pereira, M. M., and Pedrosa, J. M. 2018. "Molecular-Based Selection of Porphyrins towards the Sensing of Explosives in the Gas Phase." Sens. Actuators B 260: 116-124.

[4] Capan, I., Erdogan, M., Stanciu, G. A., Stanciu, S. G., Hristu, R., and Göktepe, M. 2012. "The Interaction between the Gas Sensing and Surface Morphology Properties of LB Thin Films of Porphyrins in Terms of the Adsorption Kinetics." Mater. Chem. Phys. 136 (2-3): 1130-1136.

[5] Yang, W., Xu, J., Mao, Y., Yang, Y., and Jiang, Y. 2016. "Detection of Volatile Organic Compounds Using Langmuir-Blodgett Films of Zinc-Porphyrin and Zinc-Phthalocyanine." Synth. React. Inorg., Met.-Org., Nano-Met. Chem. 46 (5): 735-740.

[6] Gutiérreza, A. F., Brittle, S., Richardson, T. H., and Dunbar, A. 2014. "A Proto-Type Sensor for Volatile Organic Compounds Based on Magnesium Porphyrin Molecular Films.” Sens. Actuators B 202: 854-860.

[7] Saini, R., Mahajan, A., Bedi, R. K., and Aswal, D. K. 2012. "Room Temperature Detection of Amine Vapours
Using Copper Phthalocyanine Based Thin Films.” Phys. Status Solidi A, 209 (7): 1245-1250.

[8] Gonçalves, V. C., Nunes, B. M., Balogh, D. T., and Olivati, C. A. 2010. "Detection of Volatile Organic Compounds Using a Polythiophene Derivative." Phys. Status Solidi A 207 (7): 1756-1759.

[9] Capan, İ., Tarımc1, Ç., and Capan, R. 2010. "Fabrication of Langmuir-Blodgett Thin Films of Porphyrins and Investigation on Their Gas Sensing Properties." Sens. Actuators B 144 (1): 126-130.

[10] Brittle, S. A., Richardson, T. H., Varley, L., and Hunter, L. C. A. 2010. "Amine-Sensing Properties of a Covalently Linked Calix[4]arene-porphyrin ('calixporph') Multilayered Film.” J. Porphyrins Phthalocyanines 14 (12): 1027-1033.

[11] Manera, M. G., Ferreiro-Vila, E., Cebollada, A., García-Martín, J. M., García-Martín, A., Giancane, G., Valli, L., and Rella, R. 2012. "Ethane-Bridged Zn Porphyrins Dimers in Langmuir-Schafer Thin Films: Spectroscopic, Morphologic, and Magneto-Optical Surface Plasmon Resonance Characterization.” J. Phys. Chem. C 116 (19): 10734-10742.

[12] Colombelli, A., Maneraa, M. G., Borovkov, V., Giancane, G., Valli, L., and Rella, R. 2017. "Enhanced Sensing Properties of Cobalt Bis-Porphyrin Derivative Thin Films by a Magneto-Plasmonic-Opto-Chemical Sensor." Sens. Actuators B 246: 1039-1048.

[13] Acikbas, Y., Cankaya, N., Capan, R., Erdogan, M., and Soykan, C. 2016. "Swelling Behavior of the 2-(4-methoxyphenylamino)-2-oxoethyl Methacrylate Monomer LB Thin Film Exposed to Various Organic Vapors by Quartz Crystal Microbalance Technique." J. Macromol. Sci., Part A: Pure Appl. Chem. 53 (1): 18-25.

[14] Ozmen, M., Ozbek, Z., Buyukcelebi, S., Bayrakci, M., Ertul, S., Ersoz, M., and Capan, R. 2014. "Fabrication of Langmuir-Blodgett Thin Films Of Calix[4]arenes and Their Gas Sensing Properties: Investigation of Upper Rim Para Substituent Effect." Sens. Actuators B 190: 502-511.

[15] Wilkop, T., and Ray, A. K. 2002. "Solvent Vapour Detection with A Charge Flow Transistor." J. Phys. D: Appl. Phys. 35 (20): 2661-2667. 